\title{
Mobile Learning et vidéo en Français Langue Étrangère : l'environnement matériel comme ressource en production orale
}

\section{Mobile Learning and Video in French as a Foreign Language: Using Material Environment as a Resource for Oral Production}

\author{
Laurence Martin ${ }^{1}$ a \\ ${ }^{1}$ Laboratoire Praxiling (UMR 5267), Université Paul-Valéry Montpellier \\ 34090 Montpellier, France
}

\begin{abstract}
Résumé. L'intégration des caméras dans les objets de la mobilité (ordinateur portable, appareil photo, smartphone, tablette) a conduit à l'émergence de l'autoproduction audiovisuelle comme activité sociale (YouTube) et la didactique des langues s'approprie ces nouvelles pratiques. Les apprenants peuvent se filmer facilement en dehors de la classe dans des environnements variés et transmettre leur production orale numérique à l'enseignant. En nous appuyant sur un corpus de tutoriels audiovisuels réalisés par des étudiants en français langue étrangère, nous montrerons comment les objets de la vie quotidienne participent à la construction du sens dans l'action et constituent ainsi des ressources qui aident l'apprenant à élaborer son discours et l'enseignant à évaluer la production orale. Les apprenants choisissent de se montrer en train d'agir dans des lieux qui étaient jusque-là réservés à l'apprentissage informel (salle de bain, magasin, cuisine etc.) et réalisent un produit audiovisuel en dehors de la salle de classe. L'enseignant peut intervenir sur la vidéo par exemple en repérant des erreurs dans la production orale et en insérant des annotations sur l'image. La vidéo numérique constitue une trace sur laquelle l'enseignant et l'apprenant peuvent agir de façon différée.
\end{abstract}

\begin{abstract}
The integration of cameras in the objects of mobility (laptop, camera, smartphone, tablet) led to the emergence of audiovisual self production as a social activity and the didactics of languages appropriates these new social practices. Learners can easily film themself outside the classroom in varied contexts and send their digital oral production to their teacher. Using a corpus of video tutorials produced by students in French as a foreign language, we will show how objects of everyday life participate in the construction of meaning in action and thus constitute resources shared by learners and the teacher. Learners choose to show themself in places that were previously reserved for informal learning (bathroom, kitchen, shop, etc.) and produce an audiovisual product outside the classroom. Teachers can use the
\end{abstract}

\footnotetext{
a Auteure de correspondance : laurence.martin@univ-montp3.fr
} 
video as a teaching tool by identifying learners errors and by inserting annotations on the image. Digital video then becomes a trace on which teacher and learners can act together.

\section{Introduction}

La notion de ressource est polysémique. En didactique des langues-cultures (DDLC), elle peut renvoyer au dispositif pédagogique, à des documents, des données, des outils numériques voire à des tâches pédagogiques, des personnes ou des compétences (Develotte et Pothier, 2004 ; Tomé, 2006 ; Py, 2007). Ce qui est considéré comme une ressource dans l'enseignement des langues dépend de l'évolution du contexte méthodologique institutionnel (Develotte, 2004: 11). La notion de ressource peut aussi être entendue comme l'ensemble des moyens que les co-participants mobilisent pour rendre la situation accountable (Garfinkel, 2007), c'est-à-dire descriptible : "cette descriptibilité (qui ne se confond pas avec la notion de description) est assurée par des moyens qui ne se limitent pas à la parole mais reposent sur des ressources multimodales » (Mondada, 2009: 26). C'est dans cette dernière acception que nous inscrivons notre réflexion.

En production orale, dans certaines situations, les objets participent à la construction du sens (Day, Wagner, 2014). Nous définissons un objet, à la suite de Gibson, comme « une substance persistante dotée d'une surface close ou presque close »; il a une forme, une texture et une couleur (Gibson, 2014 : 93-94). On peut le toucher, le saisir et le manipuler. L'usage d'objets dans l'apprentissage des langues est souvent limité aux objets scolaires (tableau, feutre, table, ordinateur, etc.) et peu de salle de classe sont équipées avec des objets du quotidien (comme une assiette ou un bol par exemple). Ces objets interviennent dans les activités didactiques grâce à l'image fixe ou à la vidéo (image en mouvement); ils perdent alors leur caractère ergonomique et ne sont plus saisissables, manipulables.

Dans cet article, nous montrerons comment le «tournant audiovisuel» (Stockinger, 2009) modifie la pratique de l'oral en DDLC et comment les objets sont mobilisés comme ressources par rapport à la caméra lors d'une production orale à distance et asynchrone.

\section{Cadre théorique}

\subsection{Production orale, vidéo et mobile learning en didactique des langues.}

L'étude de l'oral en français langue étrangère s'inscrit dans l'analyse des interactions en classe de langue, et mobilise la notion de cadre définie par Goffman (1974). Empruntée à Bateson, elle implique que toute activité peut avoir différents cadrages qui ne sont pas nécessairement partagés par les participants. Dans un cours de langue, les activités orales ont généralement lieu dans le cadre primaire, social et institutionnel de la salle de classe, ou dans un cadre secondaire transformé, proche de la dramaturgie (Cicurel, 2002). Des activités sociales, extérieures à la classe sont modélisées et reproduites lors de jeux de rôles, de simulations ou de jeux théâtraux. Lors du changement, des ruptures de cadre peuvent alors mettre l'apprenant en difficulté car il doit comprendre la situation de communication, imaginer un lieu et des objets puis agir dans ce cadre fictif. Les interactions orales sont certes variées mais moins spontanées (Guichon, Nicolaev, 2011: 71) que celles qui émergent dans le cadre primaire et, pour l'enseignant, l'évaluation de la cohérence entre les énoncés produits et le contexte reste problématique. Toutefois mettre à disposition des apprenants, en classe, des objets pour contextualiser chaque production orale qui a lieu dans un cadre secondaire serait difficilement envisageable notamment faute de place. L'usage de la vidéo permet alors aux apprenants de se filmer en train d'agir (cuisiner, bricoler etc.) 
avec des objets à l'extérieur de la salle de classe, dans des environnements variés. Cet usage de la vidéo dans l'enseignement- apprentissage des langues n'est pas nouveau (Compte, 1993), mais un changement dans la culture contemporaine globale est venu modifier les pratiques sociales de communication (web 2.0, peer-to-peer, réseau mobile) donnant ainsi une place importante au médium audiovisuel dans nos vies quotidiennes, privées et professionnelles ${ }^{1}$. L'usage de la vidéo s'est banalisé et «l'appropriation du langage visuel fait assister à une réinvention du quotidien » (Gunthert, $2014: 13$ ). L'intégration de ces pratiques dans l'enseignement des langues renouvelle la production orale (Li, Gromik, 2012). L'usage de la vidéo est envisagé dans une approche communic'actionnelle (Bourguignon, 2006) ; l'apprenant est un acteur social qui ne réalise pas une tâche scolaire mais une activité sociale. La caméra est un instrument (Rabardel, 1995), qui s'insère dans des pratiques sociales qui ne sont plus réservées à des groupes sociaux bien circonscrits. C'est « une entité mixte » comprenant à la fois un artefact (outil technique) et un ou des schèmes d'utilisation (Rabardel, 1995: 74). Dans cet article, nous proposons d'analyser comment, dans une pratique sociale précise, impliquant des schèmes d'utilisation spécifiques, l'usager met en image les objets pour construire du sens et rendre la situation accountable.

\subsection{Les objets dans les interactions orales}

Notre analyse de la production orale s'inscrit dans une approche anthropologique de l'activité humaine et nous empruntons des concepts formulés, en dehors de la linguistique traditionnelle. Notre recherche s'inspire de l'analyse ethnométhodologique de l'action située (Suchman, 1987 ; Hutchins, 1995), de l'analyse conversationnelle, de la cognition située (Lave, Lave\&Wenger, 1991) avec un intérêt pour le rôle des objets dans les interactions (Conein, 1994, 2005, 2014 ; Quéré, 1997 ; Relieu et al., 2004 ; Licoppe, 2008 ; Morel et Licoppe, 2012; Nevile et al., 2014). Il s'agit d'un courant de recherche pluridisciplinaire dont les concepts clés sont ceux d'《action située », de «cognition distribuée », d' " affordance », d'« embodiment», etc. (Quéré, 1997 : 165). Dans ces recherches, l'acteur partage la responsabilité de l'action avec les objets et l'espace. Mais, contrairement aux nombreux travaux sur les interactions hommes-machines, nous entendons par objet les éléments du monde physique que nous pouvons expérimenter de façon sensorielle, sans mode d'emploi car leur usage est lié à une routine c'est-à-dire « une habileté acquise par l'expérience et dont la mise en œuvre ne demande ni réflexion, ni représentation» (Quéré, 1997: 175). Pour nos analyses, nous utiliserons le concept d'affordance formulé par Gibson (1977) à la suite des travaux de Werner (1937) qui montraient déjà que les objets nous poussent à agir dans nos activités quotidiennes. Gibson construit ce néologisme à partir du verbe to afford (offrir, fournir) pour désigner une complémentarité entre l'animal et l'environnement. Ces objets sont donnés mais ils sont aussi construits dans l'interaction lorsqu'ils sont regardés, montrés, touchés, manipulés : "Their semiotic malleability and phenomenal accessibility provide an undeniable richness to talk, which we are just beginning to understand"2 (Day \& Wagner, 2014: 123). Nous souhaitons montrer ici comment les objets présents dans l'environnement sont des ressources pour l'apprenant, et pour l'enseignant, c'est-à-dire comment ils sont mobilisés pour construire du sens dans l'action.

\footnotetext{
${ }^{1}$ Voir le séminaire de Peter Stockinger (2009).

2 Traduction: «Leur mobilité sémiotique et leur accessibilité phénoménale offrent une richesse incontournable à la conversation, que nous commençons tout juste à comprendre ».
} 


\section{Méthodologie et description du corpus}

Notre recherche s'intéresse à l'activité de production orale dans un contexte institutionnel d'enseignement/apprentissage du français langue étrangère. Le terrain est celui d'une classe réunissant des étudiants du programme Erasmus, de niveau A2-B1, venus étudier en France pour quelques mois à l'université. Une première approche ethnographique du contexte, en tant qu'enseignante, nous a permis de nous rendre compte que l'évaluation finale permettant de définir le niveau en langue des étudiants accordait une place singulière à la production orale; contrairement aux autres activités de communication langagière (compréhension écrite, compréhension orale, production écrite), elle ne portait pas sur un produit fini inscrit sur un support et pouvant être annoté. Un projet audiovisuel a alors été proposé aux participants dans une démarche de recherche-action dans laquelle «le chercheur $[\ldots]$ s'intègre dans un groupe et oriente, à sa manière, le changement tout en l'enrichissant » (Duthoit, 2016). Les participants, ont eu pour tâche de réaliser chacun, en dehors du temps imparti à la formation, un tutoriel dans lequel ils devaient se mettre en scène pour présenter un savoir praxique (comme préparer un café, changer un pneu) de leur choix en interaction avec des objets (dans une cuisine, un garage etc.). Le projet consistait à échanger des savoir-faire de la vie quotidienne. Notre corpus se constitue de 17 courtes productions audiovisuelles de 2 à 5 minutes réalisées par les étudiants. Cette activité didactique était nouvelle pour l'ensemble des participants. Certains se sont filmés euxmêmes, d'autres ont fait appel à un ami proche (petit ami, colocataire etc.) mais aucun n'a rencontré de difficulté pour accomplir cette tâche. Les enregistrements ont été réalisés dans leur lieu de vie : leur studio, leur chambre, leur salle de bain et deux étudiantes ont réalisé l'enregistrement dans des magasins. Un seul étudiant a remis sa production sur une clé USB, les autres ont utilisé une application en ligne. Les 17 tutoriels créés ont été récoltés par l'enseignante en vue d'une évaluation orale individuelle qui a contribué à l'évaluation finale. Quelques vidéos qui ont été ensuite projetées en classe avec l'accord des étudiants concernés ont été annotées par l'enseignante. Des autorisations de droits à l'image ont été signées.

Les locuteurs agissent de façon verbale et corporelle, face à la caméra, dans un environnement matériel familier. Les actions verbales sont coordonnées aux actions praxiques impliquant des objets. Ces objets physiques peuvent être des objets industriels fabriqués (couteau, bouteille, robinet, cafetière, etc.), manuels ou électriques, fixes ou mobiles, des aliments conditionnés ou non, de l'eau, un corps humain ou un élément du corps (cheveux, paupières, etc.).

Nous nous intéresserons ici à une de ces vidéos, pour analyser le rôle des objets dans la mise en image et la construction du sens. Cette vidéo nous semble particulièrement intéressante car l'apprenant met en place des schèmes d'usage à des fins humoristiques. La transcription a été réalisée sans logiciel : 


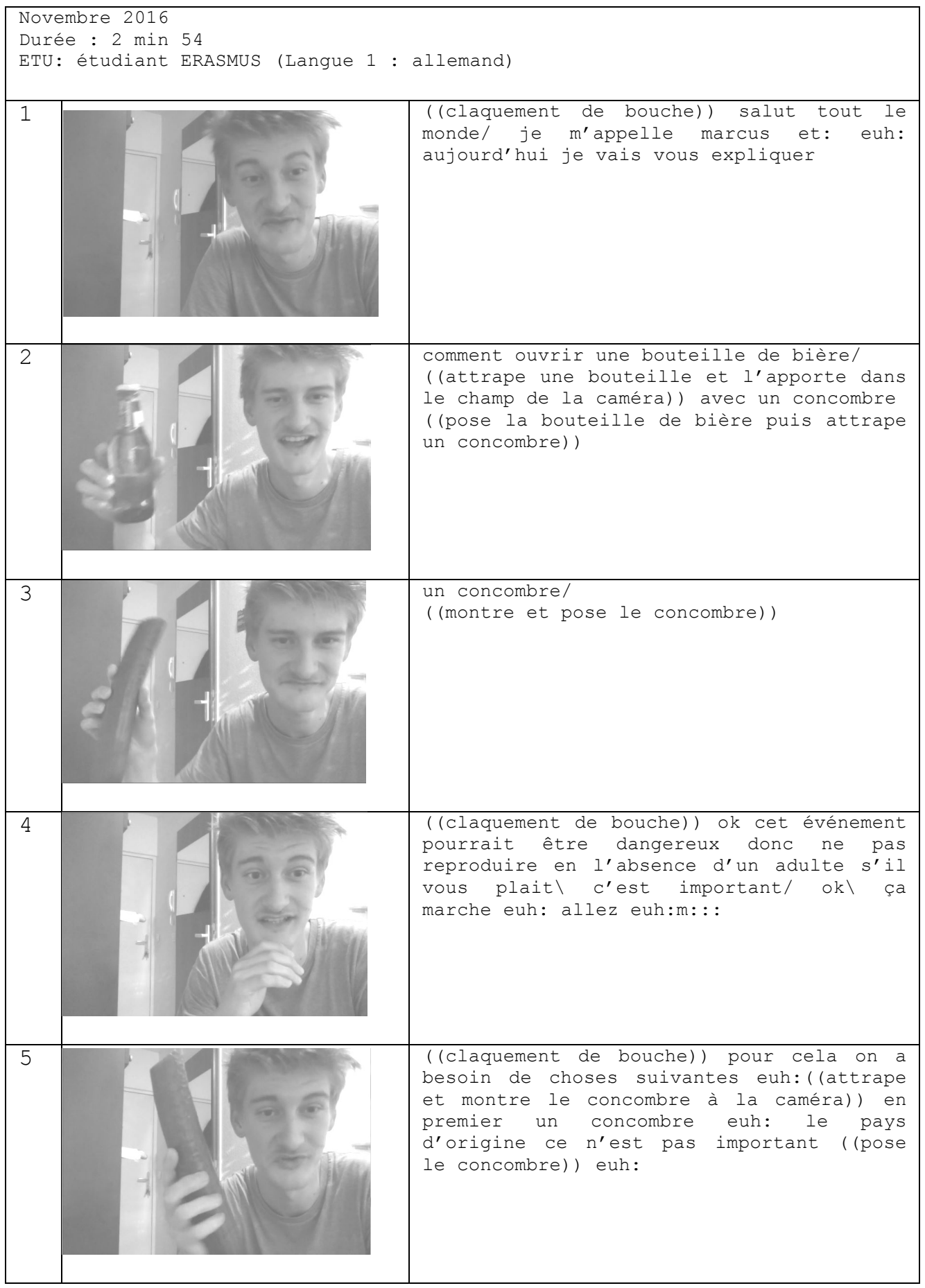




\section{ICODOC 2017}

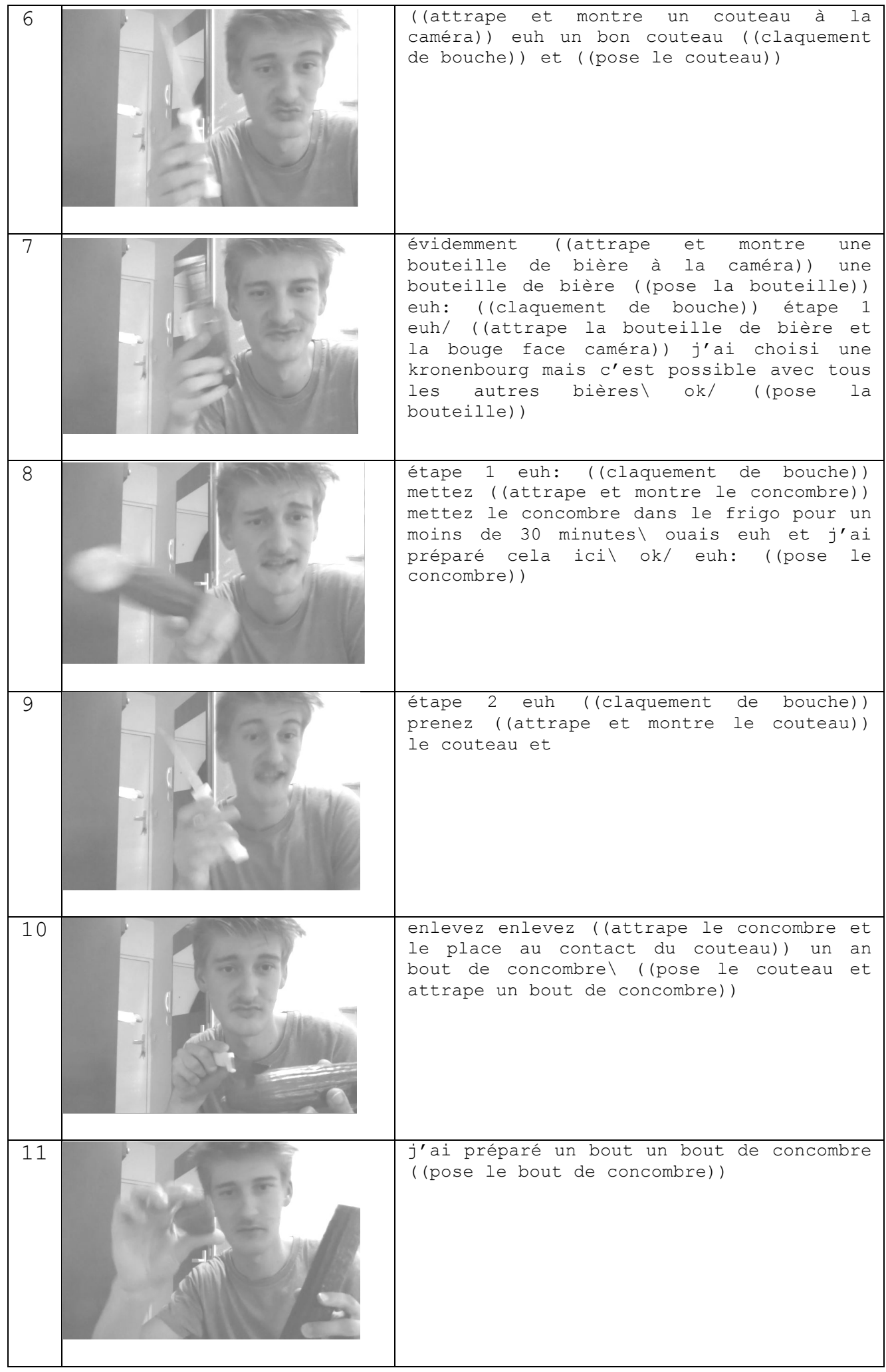




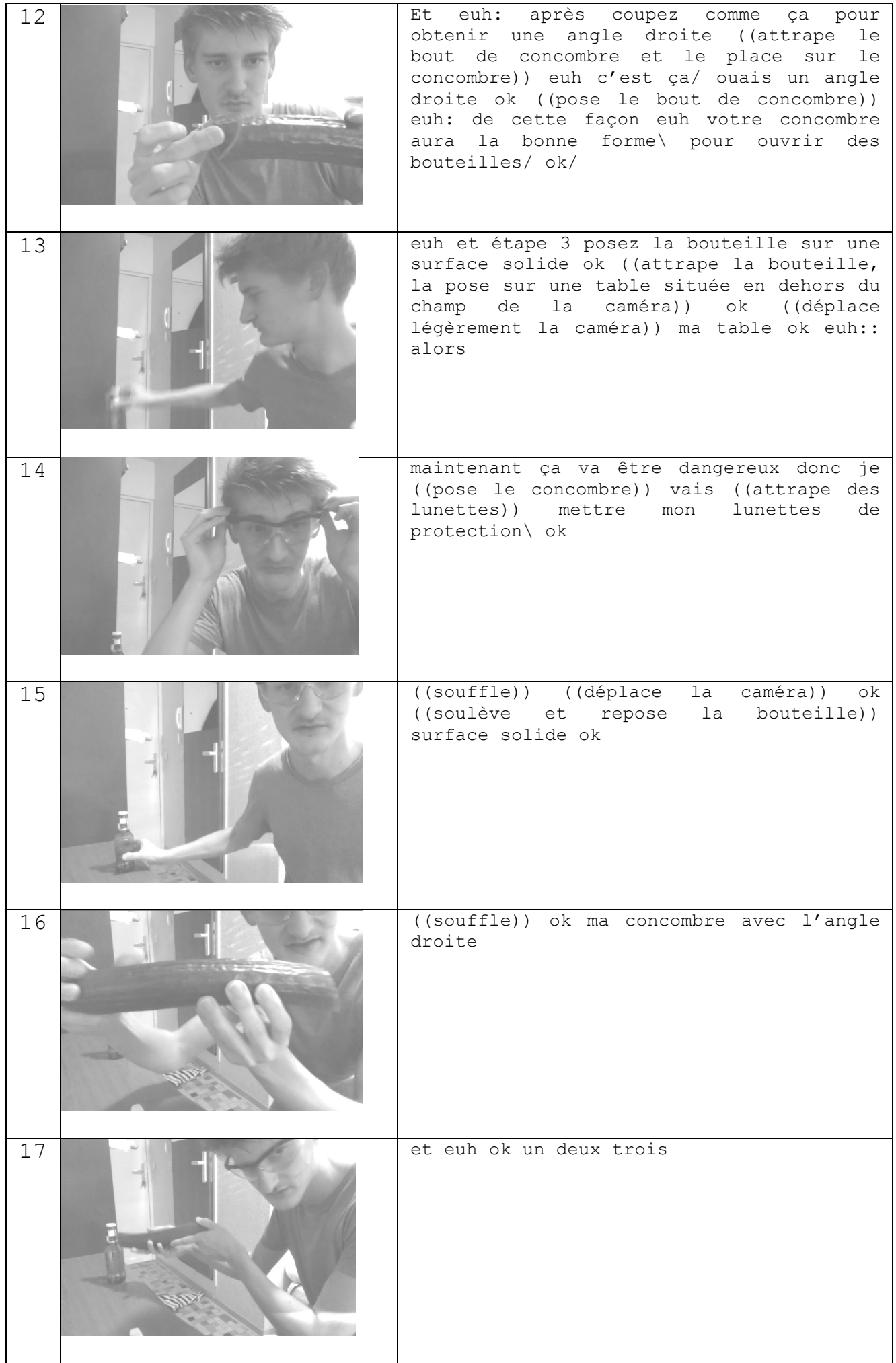




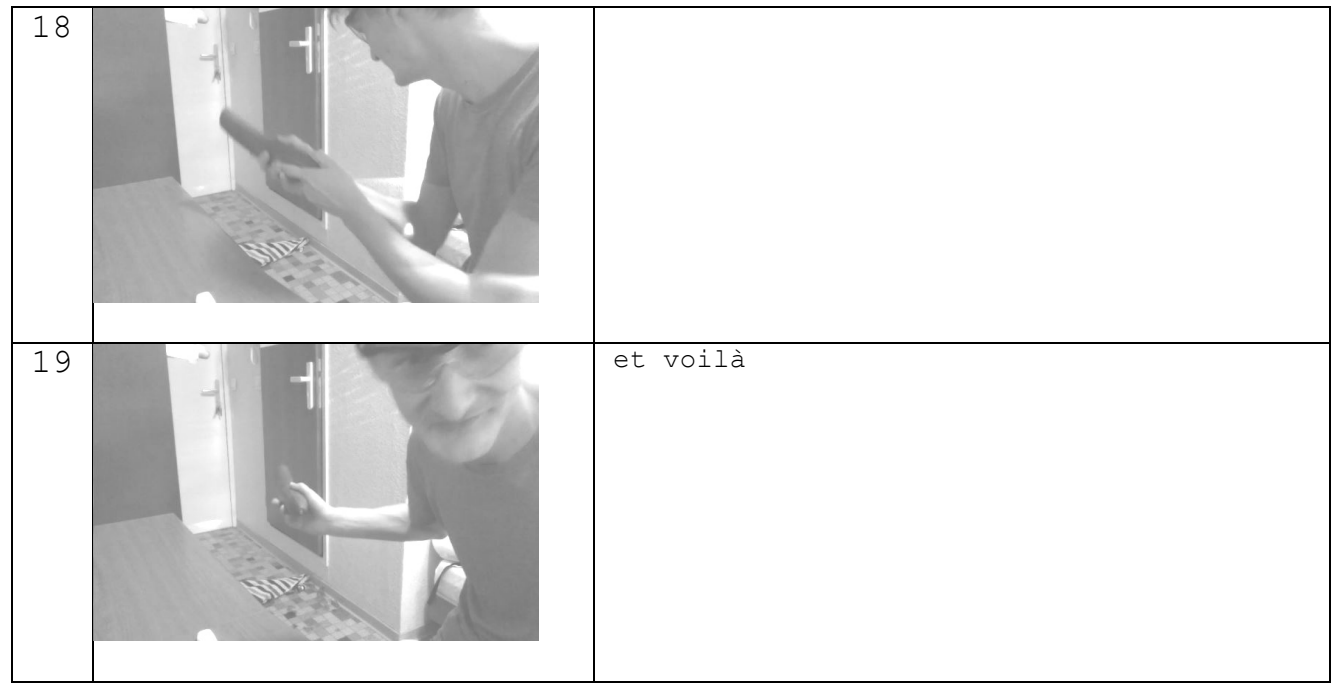

L'étudiant a choisi d'expliquer comment ouvrir une bouteille de bière avec un concombre. Le thème de sa production orale présente d'emblée un décalage avec ce qui est attendu dans un tutoriel audiovisuel. Il propose une parodie dans laquelle il s'implique corporellement.

\section{Analyses}

Le participant est face caméra dans un studio (porte et interphone, baguette de pain) et partage généralement le cadre avec des objets situés à droite dans son environnement proche. La vidéo produite est une mise en image d'une situation communicative qui se construit en relation avec l'artefact caméra. L'acteur-locuteur coordonne ses actions verbales à des actions praxiques de manipulation de divers objets pour communiquer. C'est l'annonce verbale de l'intention (1 et 2) qui capte notre attention et nous permet de saisir le sens des diverses manipulations qui vont suivre. Sans son, la situation ne serait pas descriptible ; l'acte verbal est un acte social ${ }^{3}$ de transmission de savoir-faire. La production orale est multimodale et l'accountability de la situation repose à la fois sur des ressources verbales, non verbales (mimiques, regards, postures, souffle, etc.), des gestes co-verbaux qui impliquent les mouvements des bras et des mains (pointage) $)^{4}$ et des actions praxiques (taper, casser, etc.) qui incluent des objets et leur positionnement par rapport à la caméra.

Comme dans l'étude de la gestuelle (geste iconique, métaphorique, déictique, ou de battement $)^{5}$, nous voyons que la mobilisation des objets peut avoir différentes significations. Leur manipulation peut accompagner le verbal et avoir une signification référentielle iconique, illustrative indéterminée ( « une bouteille de bière », « un concombre, 2 et 3 ) ou déterminée ( « un bon couteau », « une Kronenbourg », 6 et 7) par une forme, une marque, une qualité particulière. La présentation verbale d'un référent est généralement accompagnée de l'introduction de l'objet dans le champ de la caméra (2, 3, 5, etc.). L'apprenant agit en fonction de la caméra (postures, regards etc.) mais aussi des objets situés à proximité qu'il va mobiliser. Dès qu'il parle d'un objet mobile, il le saisit pour l'amener à l'intérieur du cadre. Il s'agit d'actes de monstration des objets (Licoppe, Morel, 2012). Cette mise en image met en valeur le caractère référentiel du verbal dans cette activité explicative.

\footnotetext{
${ }^{3}$ Et non pas un code linguistique autonome.

${ }^{4}$ Voir Di Pastena et al. (2015).

${ }^{5}$ Voir Kendon (2004) et McNeill (2000).
} 
La présence des objets donne lieu à des commentaires verbaux («le pays d'origine ce n'est pas important» ou «j'ai choisi une Kronenbourg mais c'est possible avec tous les autres bières ») qui ont ici encore un caractère comique.

La manipulation des objets peut aussi avoir une signification praxique, dans laquelle l'acteur n'illustre pas un objet mais plutôt une action impliquant un ou plusieurs objets. Les objets sont des repères dans la production verbale et leur présence physique oblige l'apprenant à employer un lexique précis (un bout, un angle droit etc.). Leur arrangement dans l'espace, qu'il a lui-même organisé (11), et leur perception dans l'action (7) guident l'apprenant dans son plan discursif. L'apprenant produit un discours verbal et corporel coordonné à l'environnement matériel. Quand il se réfère non pas à un objet mais à une action impliquant deux objets (10 et 11), il les saisit pour les mettre dans le cadre et les manipuler ensemble, c'est-à-dire l'un par rapport à l'autre. Si le nombre d'objets est supérieur, il bouge la caméra et change de cadre (17). La mise en image montre que l'acteur met le focus sur la manière d'accomplir l'action. Dans cette vidéo, le focus sur la précision des actions, renforce le caractère comique de la situation.

Des manipulations, en revanche, se substituent au langage verbal et construisent du sens à elles-seules. Elles sont souvent accompagnées de déictiques («comme ça », «voilà »). Ces actions praxiques impliquant des objets participent à l'accountability de la situation $(17,18,19)$. En effet, c'est la perception visuelle de l'action accomplie avec le concombre sur la bouteille de bière, à la fin de l'explication, qui permet de saisir le sens général de la vidéo réalisée par l'apprenant.

L'étudiant agit dans un environnement familier, un cadre secondaire qu'il a lui-même fait émerger dans un cadre primaire qu'il connaît bien (ici son propre studio) et qu'il a transformé en l'aménageant et en déclenchant la caméra en accord avec la consigne. Il s'agit en apparence d'une "modalisation» (Goffman, 1974). L'apprenant explique un savoir-faire face à la caméra pourtant il détourne ce cadre attendu et le transforme encore ; il s'agit alors d'une «fabrication » à l'initiative de l'apprenant qui « joue un tour » à son public en lui présentant une affordance insolite. L'apprenant produit des énoncés oraux situés, asynchrones et à distance. Les objets montrés et manipulés constituent des ressources cognitives situées (Conein, 1997 ; Nevile, 2014) ; ce sont des repères matériels qui participent à l'accomplissement d'un plan discursif imposé par l'intention annoncée.

L'image en mouvement présentant l'apprenant en train d'agir de façon verbale et corporelle dans un environnement matériel, c'est-à-dire de façon située, permet à l'enseignant de percevoir la cohérence entre ce que dit l'apprenant et ce qu'il fait (12). Il peut revoir la vidéo, et signaler une erreur par une annotation dans le cours de l'action en intégrant une lettre ou un symbole dans la table de montage.

\section{Conclusion}

Dans ce type de production orale à distance et asynchrone, la mise en images suggère l'importance des objets pour la compréhension partagée de la situation. Ils sont mobilisés de différentes façons ; leur manipulation permet d'illustrer le verbal, de s'y substituer, de faire émerger des commentaires sur leurs caractéristiques mais aussi d'enrichir l'explication verbale par des actions praxiques qui donnent du sens à la situation.

Leur présence contraint l'apprenant à veiller à la cohérence entre la production verbale et l'environnement matériel. La production orale est située et les objets sont mobilisés comme une ressource qui participe dans l'action à la construction du sens.

La réalisation de tutoriels audiovisuels est une pratique sociale instrumentée. Son usage dans l'enseignement/apprentissage des langues comme activité de production orale permet à l'apprenant d'agir dans un environnement situé dans un espace familier et d'engager son corps dans des actions coordonnées (verbale, co-verbale, non-verbale, praxique). 
Cette activité de production orale fait émerger une autre forme relationnelle entre l'enseignant et l'apprenant. L'apprenant choisit de se montrer en train d'interagir avec des environnements qui étaient jusque-là des environnements réservés à l'apprentissage informel (salle de bain, magasin, cuisine etc.). Il est seul responsable de ce qu'il produit. Il se présente dans un environnement public ou intime, en dehors du contexte didactique ordinaire. Il peut préparer sa production et se mettre en scène, à distance et de façon asynchrone sans affronter directement le regard de l'ensemble de ses pairs et de l'enseignant-évaluateur. Ce regard évaluateur est différé. L'apprenant a en effet la possibilité de recommencer autant de fois qu'il le souhaite puis de choisir la production orale qui sera alors diffusée, en classe ou à l'extérieur, sur une plateforme comme YouTube par exemple.

\section{Conventions de transcription}

/ \ ton montant, ton descendant

(( )) action, production vocale, description

:: allongement

\section{Bibliographie}

Bourguignon C. (2006). De l'approche communicative à l'approche communic'actionnelle : une rupture épistémologique en didactique des langues-cultures, Synergie Europe, vol. 1, p. 58-73.

Cicurel F. (2002). La classe de langue un lieu ordinaire, une interaction complexe, Acquisition et interaction en langue étrangère, vol. 16, p. 145-164.

Compte C. (1993). La vidéo en classe de langue, Paris : Hachette.

Conein B. (1997). L'action avec les objets: Un autre visage de l'action située ? Raisons pratiques, p. 25-45.

Day D. \& Wagner J. (2014). Objects as tools for talk. In M. Nevile, P. Haddington, T. Heinemann, M. Rauniomaa (Eds.) Interacting with Objects: Language, Materiality, and Social Activity. Amsterdam/Philadelphia: John Benjamins, p. 101-124.

Develotte C. (2004). Plurilinguisme et multiplicité des ressources. In C. Develotte \& M. Pothier (éds), La notion de ressources à l'heure du numérique. Lyon : ENS Éditions, p. 914.

Develotte C. et Pothier M. (éds) (2004). La notion de ressources à l'heure du numérique (Notions en question vol. 8), Lyon : ENS Éditions.

Di Pastena A., Schiaratura L. T. et Askevis-Leherpeux F. (2015). Joindre le geste à la parole : les liens entre la parole et les gestes co-verbaux, L'Année psychologique, vol. 115, no. 3, p. 463-493.

Garfinkel H. (2007). Recherches en ethnométhodologie (première parution : 1967), Paris : Presses Universitaires de France.

Gibson J. J. (1977). The Theory of Affordances. In R. Shaw \& J. Bransford (Eds.), Perceiving, Acting, and Knowing. Toward an Ecological Psychology. Hillsdale: Erlbaum, p. 127-143. 
Gibson J. J. (2014), Approche écologique de la perception visuelle (première parution: 1979), Paris : Éditions Dehors.

Goffman E. (1974). Frame Analysis: An Essay on the Organization of Experience, Cambridge, MA: Harvard University Press.

Guichon N. \& Nicolaev V. (2011). Influence de certaines caractéristiques des tâches d'apprentissage sur la production orale en L2. In E. Nissen, F. Poyer et T. Soubrié (dir.), Interagir et apprendre en ligne. Grenoble : ELLUG, p. 61-76.

Gunthert A. (2014). L'image conversationnelle. Les nouveaux usages de la photographie numérique, Études photographiques, $\mathrm{n}^{\circ} 31$

[https://journals.openedition.org/etudesphotographiques/3387].

Li J., Gromik N. \& Edwards N. (Eds.) (2012). ESL and Digital Video Integration: Case Studies, Alexandria, VA: TESOL International Association.

Licoppe C. \& Julien M. (2012). Video-in-interaction: "Talking heads" and the multimodal organization of mobile and Skype video calls, Research on Language and Social Interaction, vol. $45, \mathrm{n}^{\circ} 4$, p. 399-429.

Mondada L. (2009). La production de l'intelligibilité de l'action: une approche multimodale des procédés de sélection des locuteurs dans les interactions en classe, Éla Études de linguistique appliquée, vol. 153, p. 25-40.

Nevile M., Haddington P., Heinemann T. \& Rauniomaa M. (Eds.) (2014). Interacting with Objects: Language, Materiality, and Social Activity. Amsterdam/Philadelphia: John Benjamins.

Quéré L. (1997). La situation toujours négligée ? Réseaux, vol. 15, n 85, p. 163-192.

Rabardel P. (1995). Les hommes et les technologies; approche cognitive des instruments contemporains, Paris : Armand Colin.

Stockinger P. (2009). Analyse sémiotique du texte audiovisuel. Le tournant audiovisuel et participatif dans la culture contemporaine globale, Séminaire de Master 2009-2010, Paris : INALCO, https://hal.archives-ouvertes.fr/cel-01316383/.

Tomé M. (2006). Vers une typologie des ressources, supports et dispositifs Internet pour le français langue étrangère, Estudios humanísticos. Filología, vol. 28, p. 311-326. 